Article

\title{
Comparison of Lysis and Detachment Sample Preparation Methods for Cultured Triple-Negative Breast Cancer Cells Using UHPLC-HRMS-Based Metabolomics
}

\author{
Blake R. Rushing * ${ }^{D}$, Madison Schroder and Susan C. J. Sumner
}

check for

updates

Citation: Rushing, B.R.; Schroder, M.; Sumner, S.C.J. Comparison of Lysis and Detachment Sample Preparation Methods for Cultured Triple-Negative Breast Cancer Cells Using

UHPLC-HRMS-Based Metabolomics.

Metabolites 2022, 12, 168.

https://doi.org/10.3390/

metabo12020168

Academic Editors: Tommaso Cataldi and Cosima Damiana Calvano

Received: 30 December 2021

Accepted: 7 February 2022

Published: 10 February 2022

Publisher's Note: MDPI stays neutral with regard to jurisdictional claims in published maps and institutional affiliations.

Copyright: (c) 2022 by the authors Licensee MDPI, Basel, Switzerland. This article is an open access article distributed under the terms and conditions of the Creative Commons Attribution (CC BY) license (https:// creativecommons.org/licenses/by/ $4.0 /)$.

\author{
Department of Nutrition, Nutrition Research Institute, University of North Carolina-Chapel Hill, \\ Kannapolis, NC 28081, USA \\ * Correspondence: blake_rushing@unc.edu
}

\begin{abstract}
Dysregulation of cellular metabolism is now a well-recognized hallmark of cancer. Studies investigating the metabolic features of cancer cells have shed new light onto processes in cancer cell biology and have identified many potential novel treatment options. The advancement of mass spectrometry-based metabolomics has improved the ability to monitor multiple metabolic pathways simultaneously in various experimental settings. However, questions still remain as to how certain steps in the metabolite extraction process affect the metabolic profiles of cancer cells. Here, we use ultra-high-performance liquid chromatography-high-resolution mass spectrometry (UHPLC-HRMS) untargeted metabolomics to investigate the effects of different detachment and lysis methods on the types and abundances of metabolites extracted from MDA-MB-231 cells through the use of inhouse standards libraries and pathway analysis software. Results indicate that detachment methods (trypsinization vs. scraping) had the greatest effect on metabolic profiles whereas lysis methods (homogenizer beads vs. freeze-thaw cycling) had a lesser, though still significant, effect. No singular method was clearly superior over others, with certain metabolite classes giving higher abundances or lower variation for each detachment-lysis combination. These results indicate the importance of carefully selecting sample preparation methods for cell-based metabolomics to optimize the extraction performance for certain compound classes.
\end{abstract}

Keywords: cell metabolomics; mass spectrometry; breast cancer; pathway analysis; detachment; lysis

\section{Introduction}

Metabolic reprogramming has emerged as a hallmark of cancer, and is known to play a critical role in a multitude of biological processes, including drug resistance, activating metastasis, responding to elevated oxidative stress, adapting to hypoxic conditions, protecting against tumor-infiltrating immune cells, inducing angiogenesis, and other processes that are necessary for the survival, growth, and spread of tumors in the body [1-3]. This has led to a resurgence of interest in cancer cell metabolism to understand cancer biology and potential preventative or therapeutic options. Furthermore, it has become clear that many aspects of cancer cell metabolism can, in part, be under oncogenic control, forming a link between genetic mutations, gene expression, epigenetic alterations, and metabolism $[4,5]$. As such, studying metabolic processes in cancer research can be a powerful approach to gain a deeper understanding of the biological processes and potential therapeutic targets relevant to this disease.

For these reasons, metabolomics has become a powerful tool in cancer research. Advancements in mass spectrometry has enabled the detection of tens of thousands of spectral features in biological specimens, due to increases in sensitivity and chromatographic resolution [6]. Furthermore, the increased stability of mass accuracy and more rigorous metabolite reporting standards have allowed for the identification of more metabolites with increased 
confidence [7]. Using metabolomics to investigate the metabolic underpinnings of cancerrelated cellular mechanisms and the response to front-line treatment and new intervention strategies has increased due to the ability to simultaneously examine large numbers of pathways and metabolic drivers [8]. Challenges that remain include considerable variability in metabolic signatures that can arise from procedures used in extracting metabolites from cultured systems. Investigations have shown that methods for quenching, detachment, and cell lysis can have significant effects on the type and abundance of metabolites that can be analyzed with downstream instrumentation [9-16]. These effects can be cell line dependent, which further necessitates the need to investigate how sample preparation procedures affect extractable metabolites in different culture models [10,17].

In this study, we present the effects of two detachment methods-trypsinization and cell scraping-as well as two physical lysis methods-freeze-thaw cycling and bead homogenization - on the types and abundances of metabolites extracted from MDA-MB231 cells analyzed by ultra-high-performance liquid chromatography-high-resolution mass spectrometry (UHPLC-HRMS) (Figure 1). The MDA-MB-231 cell line is commonly used as a model of triple-negative breast cancer [18]. Using an in-house physical standards library, we describe the endogenous metabolites that were detected for the detachment and lysis methods, and present compounds belonging to a diverse set of metabolite classes/pathways in MDA-MB-231 cells. We also describe how these detachment and lysis methods affect the levels of different metabolite classes and pathways. This information will aid in allowing metabolomics researchers to choose the most appropriate sample preparation procedures for studies using this cell model, and to consider the effects of some of these processes on the metabotypes of the cells at the time of extraction.

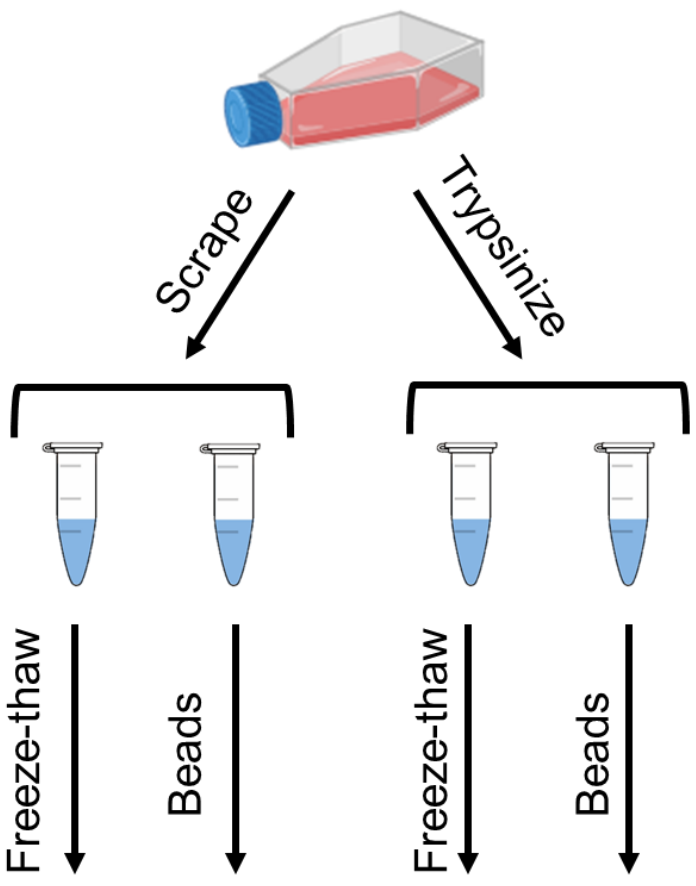

\section{Untargeted Metabolomics Analysis}

Figure 1. Sample preparation workflow. Adherent MDA-MB-231 cells were detached from flasks by either scraping or the use of trypsin. After each detachment method, cells were lysed by freeze-thaw cycling or by homogenizer beads. Cell lysates were then analyzed by UHPLC-HRMS to determine differences in metabolite abundances between the different methods. 


\section{Results}

Following normalization and preprocessing, 4479 peaks remained in the metabolomics dataset. A clear distinction between all four extraction methods can be visualized in the PCA scatter plot (Figure 2A). Supervised analyses were built to evaluate the model statistics for differentiation of the metabolite profiles for cells derived from the detachment methods or lysis methods. Model statistics indicated that both the detachment method (Figure 2B) and lysis method (Figure 2C) produced strong differences in metabolomes, as seen by the high values for R2X and R2Y and Q2 values > 0.5-a benchmark that is widely accepted for metabolomics data as an indication of reproducibility [19]. These results indicate that both detachment methods and both lysis methods each produce a distinct metabolic profile from one another.

A

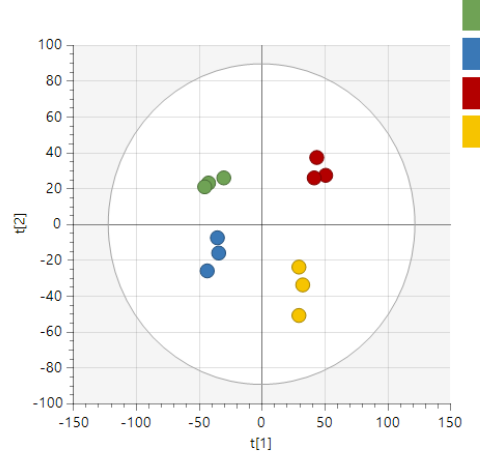

B

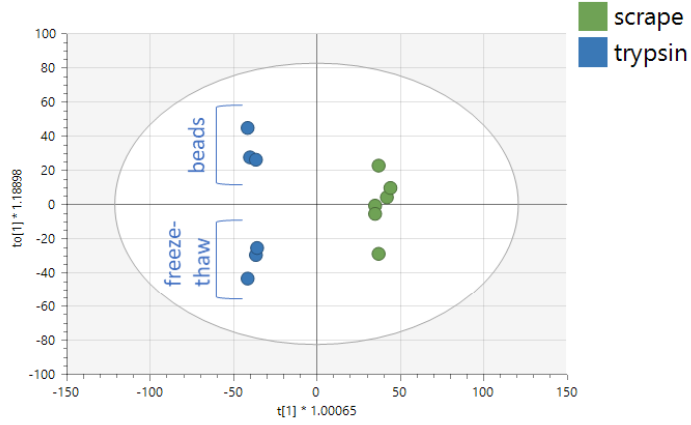

R2X: $0.53 \quad R 2 Y: 1 \quad$ Q2: 0.95

C

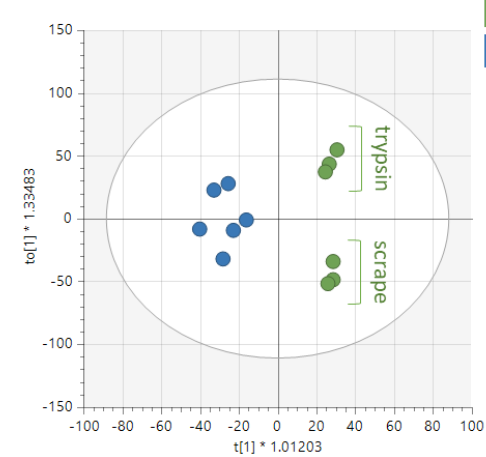

freeze-thaw

homogenizer beads scrape-freeze thaw

trypsin-freeze thaw

trypsin-homogenize scrape-homogenize

\section{$R 2 X: 0.50 \quad R 2 Y: 0.96 \quad Q 2: 0.82$}

Figure 2. Multivariate analysis of the extracted cell samples. (A) PCA of all samples showing separation of different detachment and lysis methods. (B) OPLS-DA of all trypsinized samples versus all cell-scraped samples. Sub-splitting can be seen between the bead and freeze-thaw lysis methods, particularly in the trypsinized samples. (C) OPLS-DA of all freeze-thawed samples versus all bead-homogenized samples. Sub-splitting can be seen between the bead and freeze-thaw lysis methods, particularly in the freeze-thawed samples. 
Pathway analysis was performed to identify the biochemical pathways that were significantly different between the detachment and lysis methods. Peak intensity tables were imported into MetaboAnalyst's "Functional Analysis" module to compare the trypsinized vs. scraped samples as well as homogenizer beads vs freeze-thaw cycling lysis methods. Results indicated that the detachment method (Figure 3A) perturbed a larger number of metabolic pathways as compared to the lysis method (Figure 3B). Sixteen metabolic pathways were significantly altered between the trypsinized and scraped samples and represented many different metabolite classes, such as amino acids, vitamins, sugars, and nucleotides. In contrast, the comparison between samples lysed by homogenizer beads and samples lysed by freeze-thaw cycling showed metabolic perturbations primarily in processes related to fatty acids. Pathway $p$-values for both the mummichog and GSEA algorithms, as well as the combined $p$-value, are given in Table 1 for pathways with a combined $p$-value $<0.05$.

\section{A}

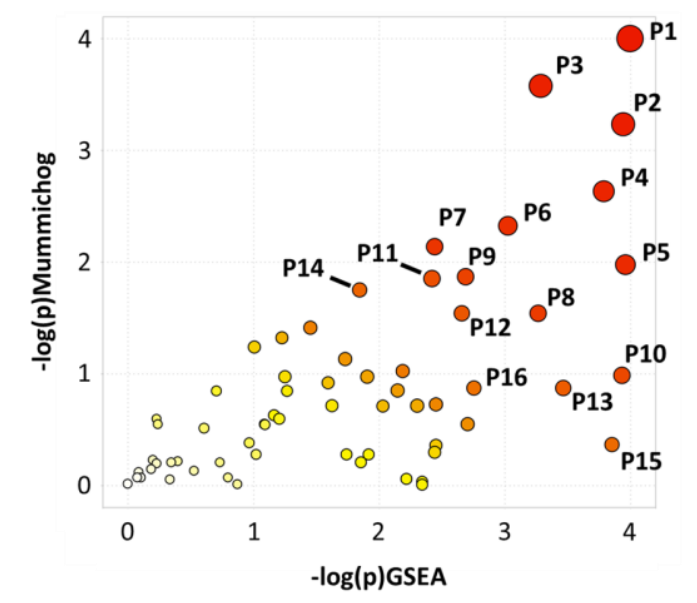

B

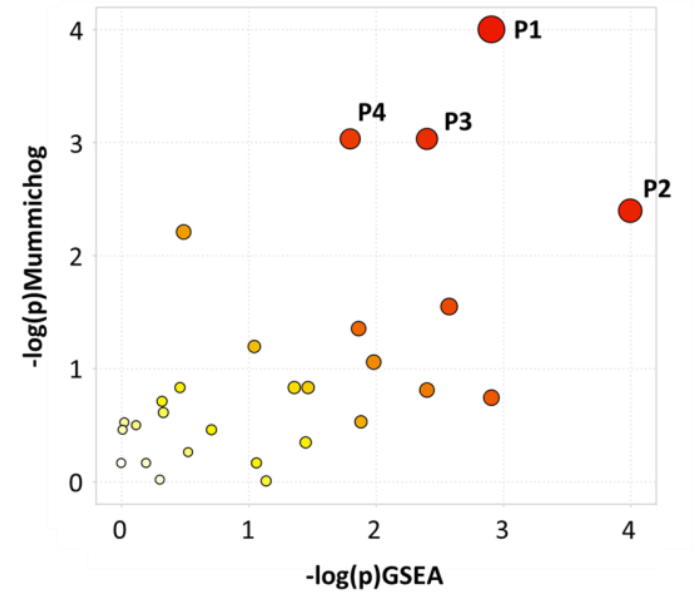

Pathway Pathway Name

Tyrosine metabolism

Urea cycle/amino group metabolism

Arginine and Proline Metabolism

Vitamin B6 (pyridoxine) metabolism

Tryptophan metabolism

Aspartate and asparagine metabolism

Vitamin B3 (nicotinate and nicotinamide) metabolism

Starch and Sucrose Metabolism

Methionine and cysteine metabolism

Glycine, serine, alanine and threonine metabolism

Alanine and Aspartate Metabolism

Nitrogen metabolism

Nucleotide Sugar Metabolism

Benzoate degradation via CoA ligation

$\mathrm{N}$-Glycan biosynthesis

Dynorphin metabolism

Figure 3. Metabolic pathways impacted by sample preparation methods. Pathway analysis was performed between (A) trypsinized vs scraped samples, and (B) freeze-thaw vs. homogenizer bead samples using MetaboAnalyst. Both the mummichog and GSEA algorithms were performed to determine the enriched pathways. Pathways with an integrated $p$-value less than 0.05 are annotated. Pathways are numbered in order of increasing integrated $p$-value.

\author{
Pathway Name \\ Fatty Acid Metabolism
}


Table 1. Significance values of the perturbed pathways due to detachment and lysis methods.

\begin{tabular}{|c|c|c|c|}
\hline \multicolumn{4}{|c|}{ Trypsinized vs. Scraped } \\
\hline Pathway Name & Mummichog_Pvals & GSEA_Pvals & Combined_Pvals \\
\hline Tyrosine metabolism & 0.00071 & 0.0101 & $9.00 \times 10^{-5}$ \\
\hline Urea cycle/amino group metabolism & 0.00285 & 0.01075 & 0.00035 \\
\hline Arginine and proline metabolism & 0.00153 & 0.02273 & 0.00039 \\
\hline Vitamin B6 (pyridoxine) metabolism & 0.00845 & 0.01282 & 0.0011 \\
\hline Tryptophan metabolism & 0.02778 & 0.01053 & 0.00267 \\
\hline Aspartate and asparagine metabolism & 0.01479 & 0.03061 & 0.00394 \\
\hline Vitamin B3 (nicotinate and nicotinamide) metabolism & 0.02075 & 0.05952 & 0.00951 \\
\hline Starch and sucrose metabolism & 0.06117 & 0.02326 & 0.01075 \\
\hline Methionine and cysteine metabolism & 0.03379 & 0.04494 & 0.01137 \\
\hline Glycine, serine, alanine and threonine metabolism & 0.1673 & 0.01087 & 0.0133 \\
\hline Alanine and aspartate metabolism & 0.03482 & 0.06098 & 0.01519 \\
\hline Nitrogen metabolism & 0.06117 & 0.04651 & 0.01952 \\
\hline Nucleotide sugar metabolism & 0.2055 & 0.01852 & 0.02501 \\
\hline Benzoate degradation via CoA ligation & 0.04187 & 0.1176 & 0.03109 \\
\hline N-Glycan biosynthesis & 0.5143 & 0.0119 & 0.03731 \\
\hline Dynorphin metabolism & 0.2055 & 0.04167 & 0.04933 \\
\hline \multicolumn{4}{|c|}{ Homogenizer Beads vs. Freeze-Thaw } \\
\hline Pathway Name & Mummichog_Pvals & GSEA_Pvals & Combined_Pvals \\
\hline Tryptophan metabolism & 0.00656 & 0.05263 & 0.0031 \\
\hline De novo fatty acid biosynthesis & 0.04632 & 0.01818 & 0.0068 \\
\hline Fatty acid activation & 0.02136 & 0.08621 & 0.01344 \\
\hline Fatty acid metabolism & 0.02136 & 0.1552 & 0.02224 \\
\hline
\end{tabular}

While the majority of peaks were higher in the scraped samples, many signals were instead higher in the trypsinized samples. In general, good reproducibility was seen among the different sample preparation methods for each peak (Figure 4). A heatmap of the top 50 identified metabolites by ANOVA $p$-value also followed this trend (Figure 5). These top 50 metabolites represent many of the significant metabolic pathways identified in the pathway analysis. In total, 99 out of the 187 metabolites identified using the in-house library at the OL1, OL2a, or OL2b ontology level had an ANOVA $p$-value $<0.05$ across the different extraction methods (Figure 6), indicating that the levels of these metabolites were significantly affected by the different sample preparation methods used in this study. Examples of metabolites with higher abundances in scraped samples include amino acids (such as histidine, leucine, phenylalanine, and glutamic acid) as well as other metabolites related to vitamin metabolism and the urea cycle (representative compounds shown in Figure 7). In contrast, trypsinized samples had higher levels of lactate, acylcarnitines, and other fatty acid-related metabolites (representative compounds shown in Figure 8). In general, the two lysis methods gave similar metabolite levels, although some acylcarnitines in the trypsinized samples were noticeably higher in the freeze-thawed samples as compared to those lysed by homogenizer beads. Table 2 provides a list of the average abundance and relative standard deviation (RSD) of all metabolites across all four methods. In terms of reproducibility, no singular method seemed to be superior to the others. Rather, each method has certain compounds with low RSD and high RSD values. Table S1 displays the variation of each named metabolite across the quality control study pool (QCSP) injections. Greater than $90 \%$ of the named metabolites had an RSD less than $30 \%$ across the QCSP injections, indicating sufficient analytical reproducibility for this study. 


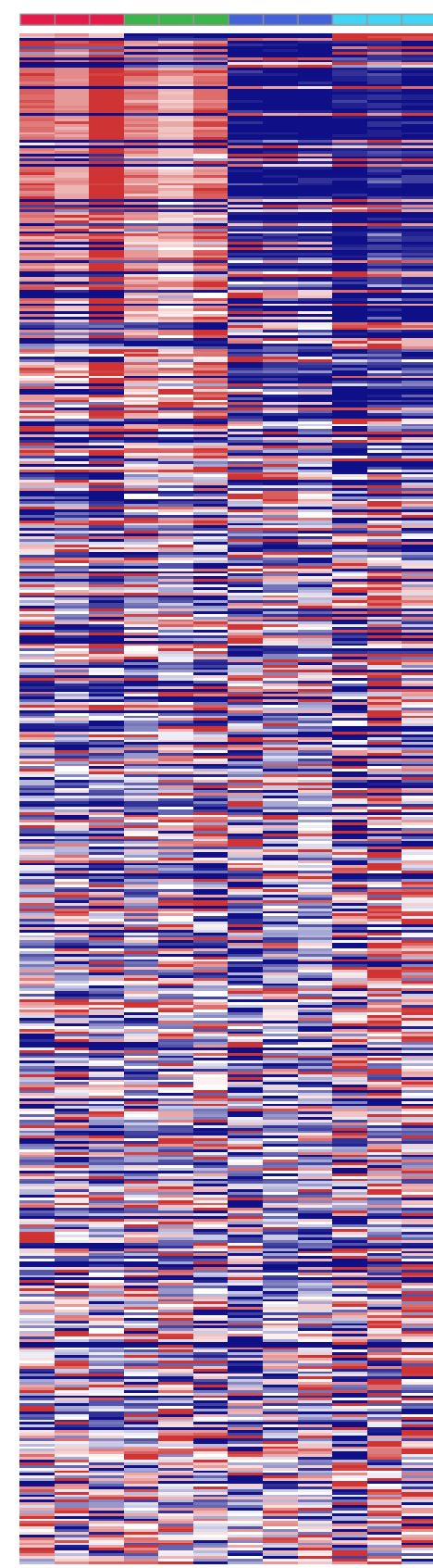

class

Scrape-freeze thaw

Scrape-homogenize

Trypsinize-homogenize

Tryspinisze-freeze thaw

Figure 4. Heatmap of all metabolomics peaks. All peaks were imported into MetaboAnalyst to visualize the peak abundances across different sample preparation methods. The detachment method showed the largest difference in metabolome profiles as compared to the lysis method. 


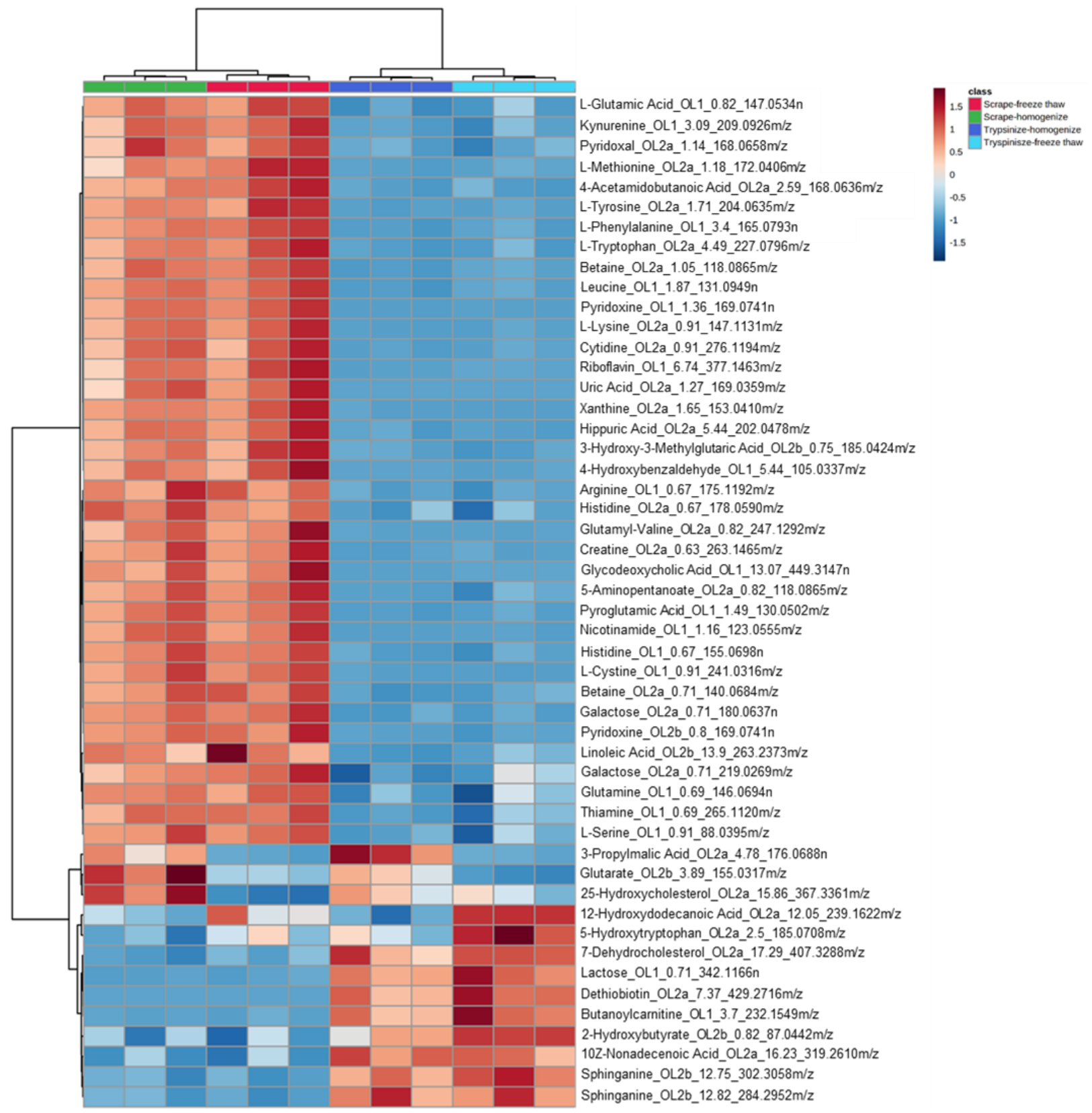

Figure 5. Heatmap of the top 50 metabolites by ANOVA $p$-value. Signals matched to the inhouse library were input into MetaboAnalyst's "Statistical Analysis" module to generate a heatmap across the different sample preparation methods. Metabolites are displayed in the following format: compound name_ontology level_retention time_mass. Masses ending with $n$ are neutral masses whereas masses ending in $m / z$ are ion masses. 
- Significant(99) O Unsignificant(88)

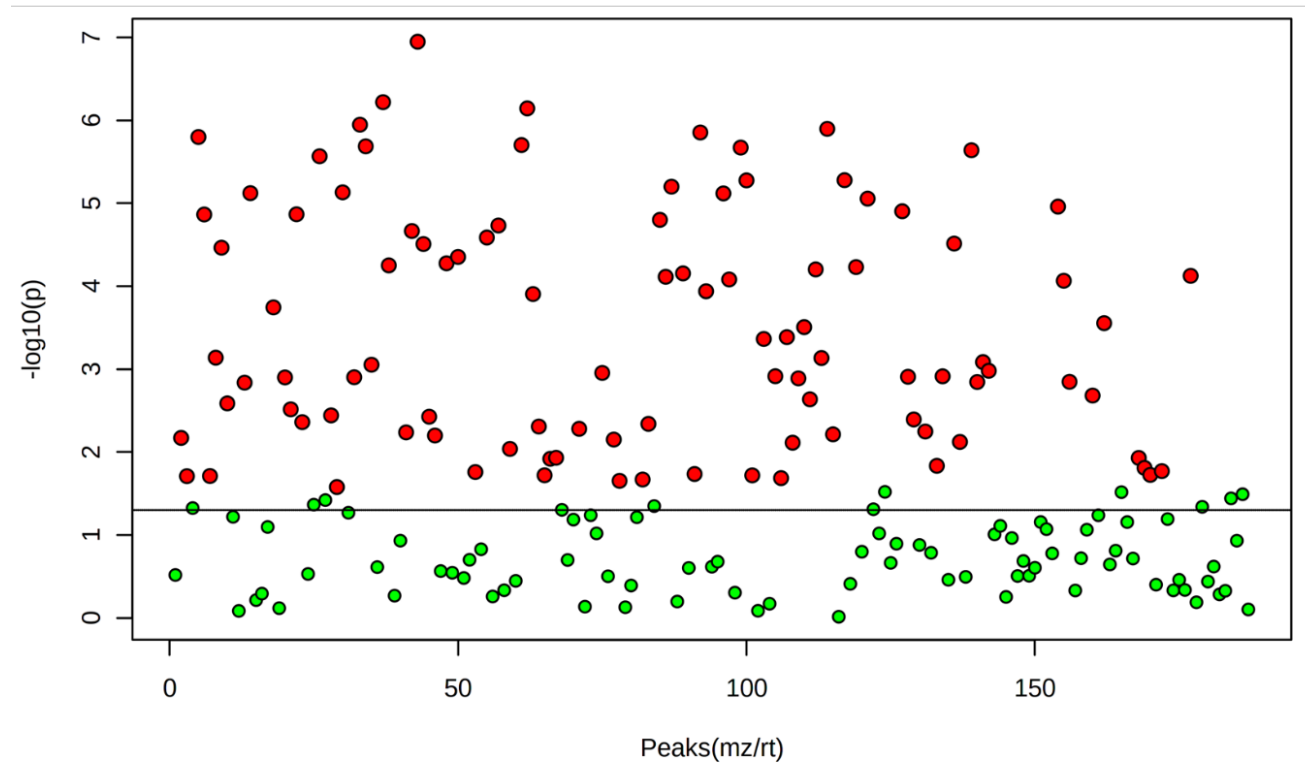

Figure 6. ANOVA scatter plot of the identified metabolites across each extraction method. Each point on the scatter plot represents one signal that matched to the in-house library of standards.
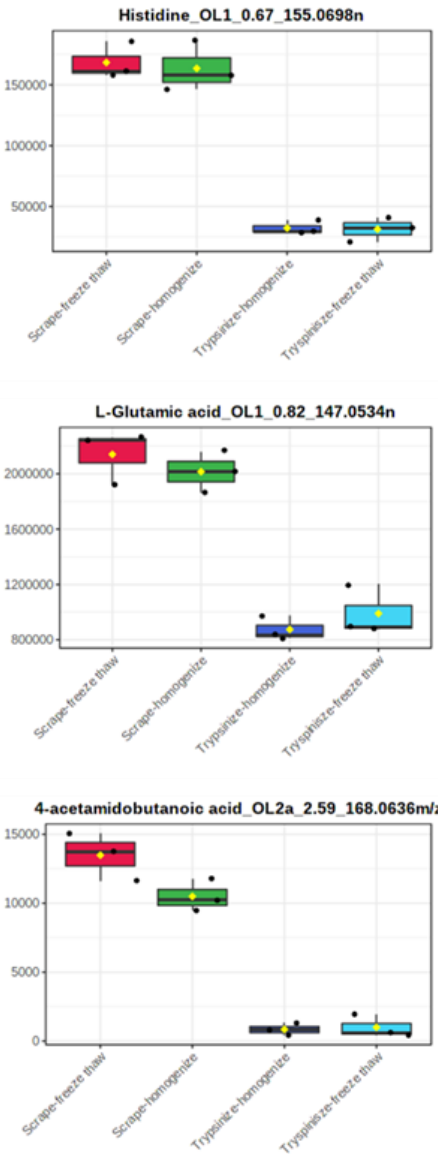
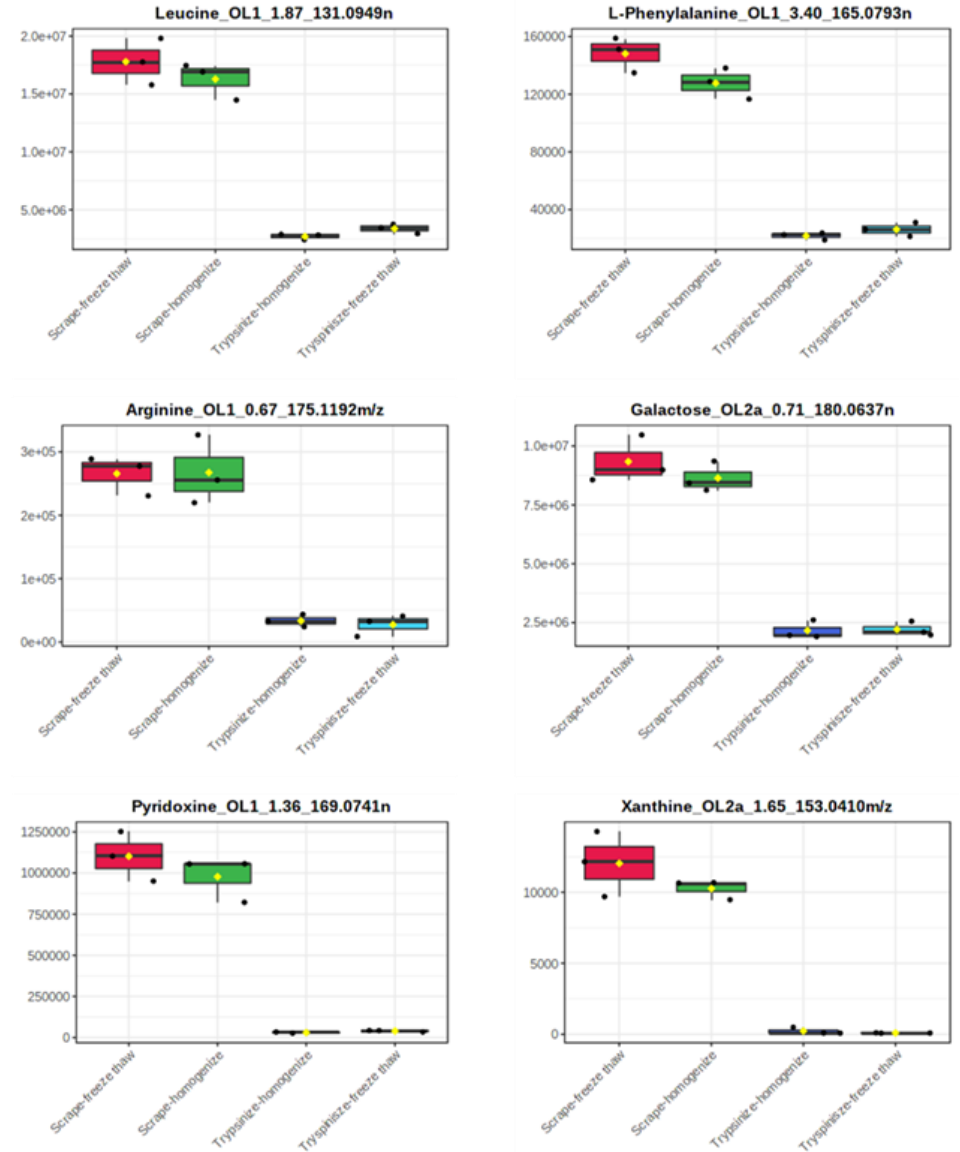

Figure 7. Representative metabolites that were increased in the scraped samples. Metabolites are displayed in the following format: compound name_ontology level_retention time_mass. Masses ending with $n$ are neutral masses whereas masses ending in $m / z$ are ion masses. 

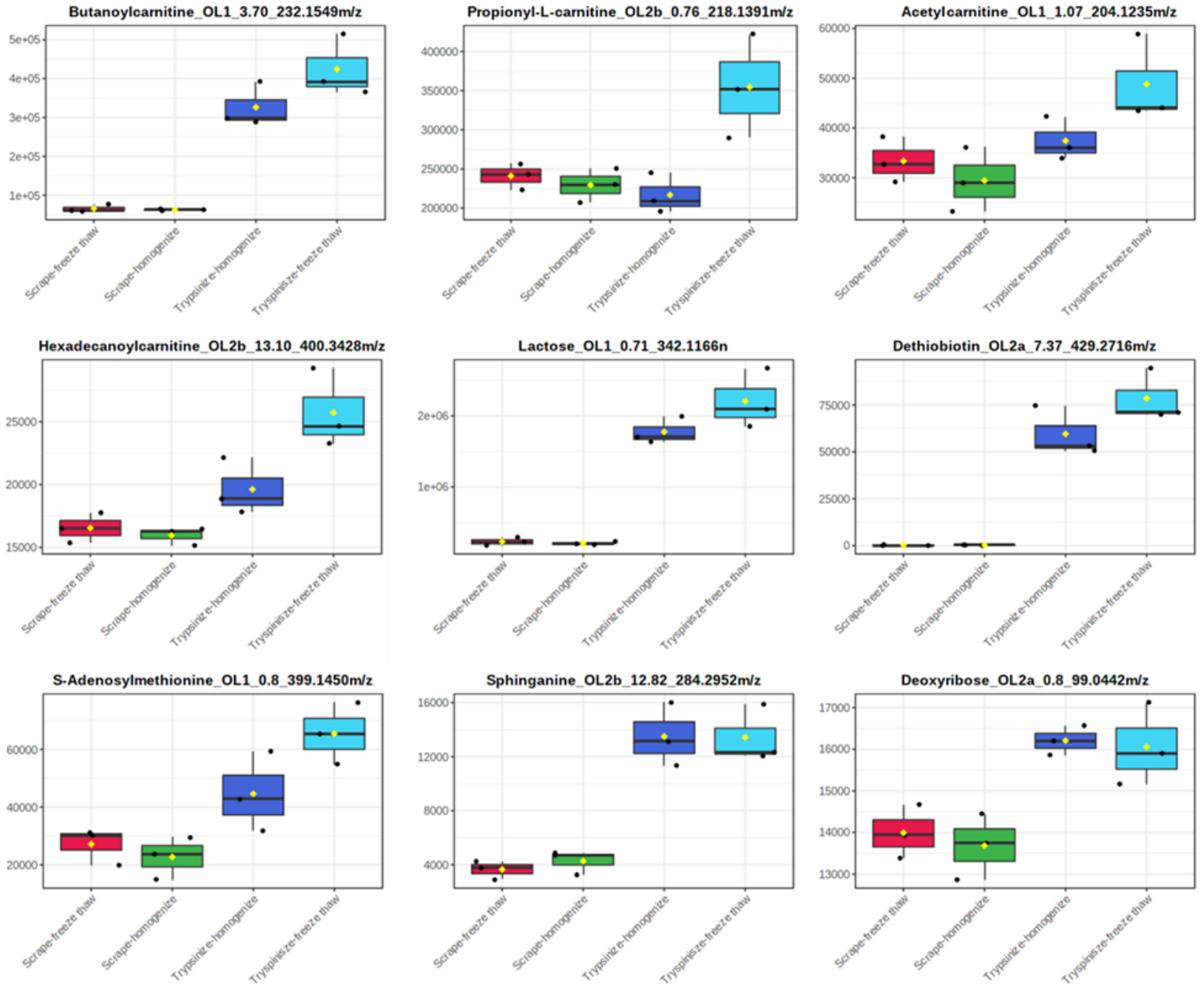

Figure 8. Representative metabolites that were increased in trypsinized samples. Metabolites are displayed in the following format: compound name_ontology level_retention time_mass. Masses ending with $n$ are neutral masses whereas masses ending in $m / z$ are ion masses. 
Table 2. Average intensities and relative standard deviations for the identified peaks.

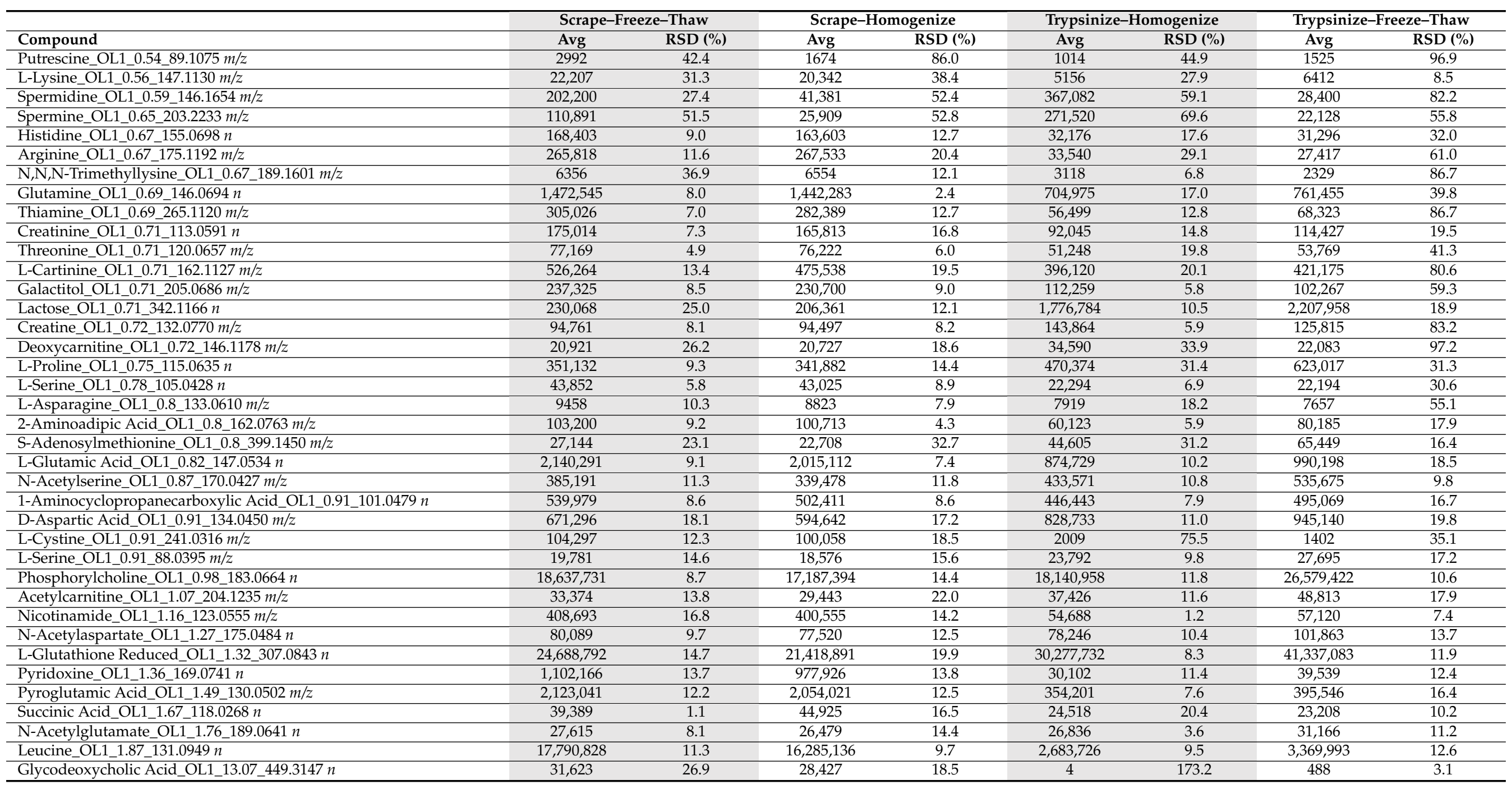


Table 2. Cont.

\begin{tabular}{|c|c|c|c|c|c|c|c|c|}
\hline \multirow[b]{2}{*}{ Compound } & \multicolumn{2}{|c|}{ Scrape-Freeze-Thaw } & \multicolumn{2}{|c|}{ Scrape-Homogenize } & \multicolumn{2}{|c|}{ Trypsinize-Homogenize } & \multicolumn{2}{|c|}{ Trypsinize-Freeze-Thaw } \\
\hline & Avg & RSD (\%) & Avg & RSD (\%) & Avg & RSD (\%) & Avg & RSD (\%) \\
\hline Palmitoleic Acid_OL1_13.6_254.2250 n & 45,654 & 4.2 & 48,022 & 4.4 & 49,662 & 5.6 & 47,474 & 10.8 \\
\hline Propanoylcarnitine_OL1_2.17_218.1391 m/z & 143,866 & 12.8 & 128,195 & 16.4 & 108,344 & 13.1 & 147,557 & 14.2 \\
\hline Adenosine_OL1_2.62_268.1045 m/z & 322,597 & 14.4 & 234,125 & 23.3 & 157,091 & 65.4 & 64,987 & 16.1 \\
\hline Kynurenine_OL1_3.09_209.0926 m/z & 8736 & 13.0 & 7779 & 18.4 & 1318 & 15.1 & 1321 & 69.8 \\
\hline L-Phenylalanine_OL1_3.4_165.0793 $\mathrm{n}$ & 148,133 & 8.3 & 127,736 & 8.3 & 21,789 & 11.4 & 26,215 & 18.3 \\
\hline Butanoylcarnitine_OL1_3.7_232.1549 m/z & 65,075 & 15.5 & 62,277 & 3.8 & 326,341 & 17.7 & 424,502 & 18.7 \\
\hline Pantothenate_OL1_4.16_219.1112 $n$ & $2,002,286$ & 5.7 & $1,819,190$ & 15.1 & $1,150,498$ & 10.8 & $1,601,215$ & 13.8 \\
\hline Valerylcarnitine_OL1_4.86_246.1705 m/z & 87,542 & 11.4 & 76,800 & 19.6 & 43,760 & 17.4 & 58,454 & 19.0 \\
\hline Methylthioadenosine_OL1_4.95_298.0974 $\mathrm{m} / \mathrm{z}$ & 247,549 & 7.6 & 247,579 & 11.7 & 203,744 & 19.4 & 220,645 & 13.0 \\
\hline 2-Hydroxyphenylacetate_OL1_6.47_153.0549 m/z & 12,314 & 4.1 & 13,598 & 9.1 & 11,840 & 4.3 & 11,954 & 14.8 \\
\hline Riboflavin_OL1_6.74_377.1463 m/z & 45,334 & 21.5 & 36,882 & 24.1 & 590 & 27.5 & 1343 & 24.9 \\
\hline 2-Octenedioic Acid_OL1_7.13_173.0811 m/z & 5113 & 44.6 & 7319 & 57.7 & 2395 & 51.0 & 4038 & 92.4 \\
\hline Azelate_OL1_8.7_188.1053 n & 268,336 & 2.9 & 253,718 & 4.8 & 253,716 & 2.9 & 288,027 & 12.8 \\
\hline Lumichrome_OL1_8.72_265.0701 m/z & 3870 & 22.1 & 4448 & 14.0 & 2179 & 26.4 & 1606 & 89.5 \\
\hline Glycerol_OL2a_0.6_115.0367 m/z & 1121 & 32.7 & 2066 & 10.9 & 1477 & 31.3 & 2534 & 50.0 \\
\hline Creatine_OL2a_0.63_263.1465 m/z & 75,190 & 21.2 & 70,905 & 20.0 & 1227 & 113.3 & 2808 & 59.6 \\
\hline N,N,N-Trimethyllysine_OL2a_0.65_211.1420 m/z & 577 & 123.1 & 287 & 173.2 & 0 & - & 373 & 90.5 \\
\hline Histidine_OL2a_0.67_178.0590 m/z & 12,284 & 7.7 & 13,639 & 8.6 & 3812 & 31.7 & 3235 & 60.2 \\
\hline Sarcosine_OL2a_0.69_112.0370 m/z & 868 & 32.3 & 742 & 27.9 & 1370 & 7.2 & 1159 & 81.7 \\
\hline Betaine_OL2a_0.71_140.0684 m/z & 104,380 & 9.4 & 94,252 & 13.8 & 16,865 & 21.0 & 21,989 & 21.6 \\
\hline Galactose_OL2a_0.71_180.0637 $n$ & $9,339,956$ & 10.7 & $8,638,770$ & 7.4 & $2,165,989$ & 18.2 & $2,210,381$ & 14.0 \\
\hline Galactose_OL2a_0.71_219.0269 m/z & 888,396 & 7.0 & 783,629 & 6.6 & 376,622 & 18.2 & 529,905 & 20.4 \\
\hline Trimethylamine Oxide_OL2a_0.71_76.0758 m/z & 624 & 52.7 & 815 & 32.0 & 105 & 62.9 & 31 & 173.2 \\
\hline Arabitol_OL2a_0.72_175.0580 m/z & 8611 & 47.2 & 8464 & 15.0 & 3476 & 13.8 & 2267 & 86.6 \\
\hline Sorbitol_OL2a_0.72_221.0425 m/z & 14,185 & 8.6 & 13,373 & 2.7 & 6125 & 21.6 & 6555 & 80.8 \\
\hline Sucrose_OL2a_0.72_325.1133 $\mathrm{m} / \mathrm{z}$ & 520 & 173.2 & 420 & 173.2 & 6975 & 17.8 & 4244 & 89.9 \\
\hline Pipecolate_OL2a_0.72_94.0653 m/z & 3490 & 27.8 & 5576 & 16.4 & 6494 & 53.1 & 1542 & 57.2 \\
\hline Uracil_OL2a_0.75_113.0347 m/z & 3350 & 30.5 & 3567 & 9.9 & 6473 & 53.9 & 3620 & 13.5 \\
\hline N-Acetylputrescine_OL2a_0.75_113.1075 m/z & 0 & - & 160 & 173.2 & 607 & 70.5 & 21 & 173.2 \\
\hline Glycerol_OL2a_0.75_115.0368 m/z & 6206 & 50.0 & 6747 & 12.6 & 1357 & 32.5 & 1147 & 93.2 \\
\hline Creatine_OL2a_0.75_154.0589 $\mathrm{m} / \mathrm{z}$ & 3381 & 48.7 & 4280 & 19.9 & 3520 & 4.8 & 2818 & 87.7 \\
\hline Deoxyribose_OL2a_0.76_117.0548 m/z & 11,747 & 15.8 & 10,872 & 43.0 & 4871 & 14.0 & 5724 & 63.0 \\
\hline Choline_OL2a_0.76_143.0705 m/z & 3740 & 10.7 & 3714 & 7.9 & 4828 & 12.2 & 3992 & 17.7 \\
\hline Taurine_OL2a_0.78_126.0221 m/z & 7535 & 30.2 & 7801 & 24.1 & 68,493 & 22.3 & 71,492 & 38.9 \\
\hline 3-(Carbamoylamino)Propanoic Acid_OL2a_0.78_155.0429 m/z & 4671 & 28.5 & 4045 & 43.0 & 7551 & 2.6 & 6199 & 65.8 \\
\hline Pyridoxal_OL2a_0.78_168.0658 m/z & 1977 & 18.9 & 2218 & 36.2 & 917 & 14.3 & 518 & 72.0 \\
\hline
\end{tabular}


Table 2. Cont.

\begin{tabular}{|c|c|c|c|c|c|c|c|c|}
\hline \multirow[b]{2}{*}{ Compound } & \multicolumn{2}{|c|}{ Scrape-Freeze-Thaw } & \multicolumn{2}{|c|}{ Scrape-Homogenize } & \multicolumn{2}{|c|}{ Trypsinize-Homogenize } & \multicolumn{2}{|c|}{ Trypsinize-Freeze-Thaw } \\
\hline & Avg & RSD (\%) & Avg & RSD (\%) & Avg & RSD (\%) & Avg & RSD (\%) \\
\hline Fructose_OL2a_0.78_213.0973 m/z & 181 & 87.8 & 59 & 173.2 & 3964 & 15.8 & 3376 & 87.2 \\
\hline Glycine_OL2a_0.78_76.0394 m/z & 16,396 & 6.1 & 15,563 & 7.8 & 17,457 & 11.3 & 18,217 & 31.4 \\
\hline 5-Aminolevulinate_OL2a_0.8_132.0657 m/z & 7413 & 13.8 & 8389 & 15.6 & 7112 & 10.4 & 6998 & 15.0 \\
\hline 3-Methyladenine_OL2a_0.8_150.0777 m/z & 1058 & 13.8 & 1531 & 7.9 & 11,761 & 78.6 & 1813 & 30.9 \\
\hline Spermine_OL2a_0.8_203.2234 m/z & 71,359 & 30.7 & 41,512 & 20.4 & 183,518 & 57.7 & 11,527 & 48.6 \\
\hline Deoxyribose_OL2a_0.8_99.0442 m/z & 13,993 & 4.7 & 13,684 & 5.7 & 16,206 & 2.2 & 16,056 & 6.2 \\
\hline Xylose_OL2a_0.82_115.0392 m/z & 7632 & 15.1 & 7779 & 7.4 & 9921 & 11.4 & 10,416 & 16.3 \\
\hline 5-Aminopentanoate_OL2a_0.82_118.0865 m/z & 722,647 & 13.1 & 669,054 & 13.3 & 187,580 & 3.1 & 193,015 & 31.4 \\
\hline Glutamyl-Valine_OL2a_0.82_247.1292 $\mathrm{m} / \mathrm{z}$ & 20,682 & 27.9 & 18,403 & 19.8 & 70 & 173.2 & 53 & 173.2 \\
\hline Dihydroorotic Acid_OL2a_0.91_191.0666 m/z & 7007 & 15.9 & 6597 & 37.0 & 7747 & 11.6 & 5671 & 47.1 \\
\hline Cytidine_OL2a_0.91_276.1194 m/z & 136,179 & 23.1 & 126,828 & 18.5 & 16,521 & 10.8 & 19,062 & 15.1 \\
\hline Hypotaurine_OL2a_0.96_110.0272 m/z & 2926 & 14.9 & 2486 & 13.9 & 2760 & 15.6 & 3432 & 23.4 \\
\hline Phosphorylcholine_OL2a_0.96_183.0664 $n$ & 162,563 & 29.0 & 132,261 & 47.2 & 135,874 & 40.8 & 391,675 & 37.4 \\
\hline Betaine_OL2a_1.05_118.0865 m/z & $1,157,973$ & 9.1 & $1,053,217$ & 12.3 & 268,966 & 3.9 & 311,624 & 6.2 \\
\hline Pyridoxal_OL2a_1.14_168.0658 m/z & 3417 & 15.3 & 3279 & 22.2 & 763 & 21.0 & 643 & 55.8 \\
\hline 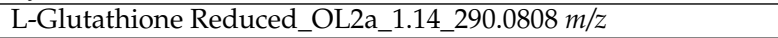 & 1975 & 49.5 & 1679 & 49.3 & 2154 & 16.0 & 6728 & 92.6 \\
\hline Shikimic Acid_OL2a_1.16_139.0392 m/z & 1048 & 16.7 & 1247 & 6.5 & 887 & 26.9 & 1004 & 22.1 \\
\hline L-Methionine_OL2a_1.18_172.0406 m/z & 8726 & 14.2 & 6260 & 22.7 & 173 & 87.8 & 396 & 57.8 \\
\hline Uric Acid_OL2a_1.27_169.0359 m/z & 5373 & 18.1 & 4778 & 25.6 & 0 & - & 0 & - \\
\hline Xanthine_OL2a_1.65_153.0410 m/z & 12,029 & 19.1 & 10,254 & 6.7 & 165 & 173.2 & 0 & - \\
\hline L-Tyrosine_OL2a_1.71_204.0635 m/z & 23,973 & 19.5 & 20,174 & 8.0 & 1793 & 19.2 & 2099 & 18.7 \\
\hline Sebacate_OL2a_10.06_225.1102 m/z & 92,992 & 5.6 & 92,015 & 3.1 & 92,176 & 2.8 & 103,904 & 4.8 \\
\hline $\begin{array}{l}\text { 2-(2-Carboxyethyl)-4-Methyl-5-Propylfuran-3-Carboxylic Acid } \\
\text { (CMPF)_OL2a_11.97_263.0895 m/z }\end{array}$ & 3580 & 12.5 & 3631 & 11.5 & 3347 & 12.9 & 3506 & 6.4 \\
\hline 12-Hydroxydodecanoic Acid_OL2a_12.05_239.1622 m/z & 300,056 & 5.7 & 276,373 & 2.7 & 266,376 & 3.2 & 326,278 & 0.1 \\
\hline 17-Octadecynoic Acid_OL2a_14.74_303.2296 m/z & 3451 & 17.0 & 3669 & 23.0 & 3747 & 10.7 & 4109 & 16.9 \\
\hline Oleic Acid_OL2a_15.49_283.2634 m/z & 4171 & 39.5 & 9314 & 5.9 & 12,433 & 23.0 & 5478 & 6.8 \\
\hline Oleic Acid_OL2a_15.67_283.2635 m/z & 716 & 106.3 & 2885 & 35.4 & 2845 & 44.9 & 538 & 87.0 \\
\hline 25-Hydroxycholesterol_OL2a_15.86_367.3361 m/z & 5383 & 28.5 & 31,189 & 14.6 & 21,320 & 21.8 & 15,555 & 31.2 \\
\hline Arachidonic Acid_OL2a_15.88_287.2371 m/z & 0 & - & 692 & 97.0 & 3528 & 43.2 & 1462 & 46.3 \\
\hline 24-Hydroxychloesterol_OL2a_16.12_425.3393 m/z & 2979 & 51.4 & 6453 & 25.3 & 8977 & 14.7 & 12,584 & 20.6 \\
\hline 10Z-Nonadecenoic Acid_OL2a_16.23_319.2610 m/z & 1139 & 119.5 & 1296 & 72.6 & 6095 & 12.4 & 5706 & 15.5 \\
\hline 10Z-Nonadecenoic Acid_OL2a_16.25_297.2792 m/z & 895 & 117.9 & 206 & 173.2 & 3779 & 21.6 & 2407 & 32.8 \\
\hline 7-Dehydrocholesterol_OL2a_17.29_407.3288 m/z & 2855 & 22.6 & 1802 & 35.4 & 9337 & 27.6 & 11,165 & 1.8 \\
\hline 5-Hydroxytryptophan_OL2a_2.5_185.0708 m/z & 2510 & 16.1 & 1877 & 15.5 & 2485 & 16.8 & 3953 & 8.6 \\
\hline 4-Acetamidobutanoic Acid_OL2a_2.59_168.0636 m/z & 13,487 & 12.8 & 10,492 & 11.3 & 847 & 51.9 & 998 & 83.1 \\
\hline Cyclic AMP_OL2a_3.04_330.0603 m/z & 755 & 39.1 & 914 & 64.0 & 10,860 & 3.2 & 8639 & 69.4 \\
\hline
\end{tabular}


Table 2. Cont.

\begin{tabular}{|c|c|c|c|c|c|c|c|c|}
\hline \multirow[b]{2}{*}{ Compound } & \multicolumn{2}{|c|}{ Scrape-Freeze-Thaw } & \multicolumn{2}{|c|}{ Scrape-Homogenize } & \multicolumn{2}{|c|}{ Trypsinize-Homogenize } & \multicolumn{2}{|c|}{ Trypsinize-Freeze-Thaw } \\
\hline & Avg & RSD (\%) & Avg & RSD (\%) & Avg & RSD (\%) & Avg & RSD (\%) \\
\hline Methyglutarate_OL2a_4.49_169.0475 m/z & 11,990 & 5.5 & 11,958 & 6.8 & 11,634 & 4.9 & 11,874 & 13.4 \\
\hline L-Tryptophan_OL2a_4.49_227.0796 m/z & 45,154 & 14.4 & 38,462 & 10.7 & 7886 & 12.8 & 8910 & 30.9 \\
\hline Tiglylcarnitine_OL2a_4.59_244.1548 m/z & 6477 & 4.4 & 5385 & 13.6 & 4495 & 3.9 & 3982 & 86.8 \\
\hline 3-Propylmalic Acid_OL2a_4.78_176.0688 n & 8328 & 20.2 & 46,028 & 22.2 & 65,600 & 19.5 & 9590 & 11.2 \\
\hline 3-Hydroxysuberic Acid_OL2a_5.2_173.0812 $\mathrm{m} / \mathrm{z}$ & 1098 & 61.7 & 761 & 54.0 & 337 & 56.2 & 362 & 51.2 \\
\hline Hippuric Acid_OL2a_5.44_202.0478 m/z & 20,814 & 18.4 & 18,500 & 13.1 & 2256 & 31.7 & 2030 & 10.9 \\
\hline 3,3-Dimethylpentanedionate_OL2a_6.08_183.0632 $\mathrm{m} / \mathrm{z}$ & 26,837 & 7.0 & 25,321 & 8.2 & 22,685 & 1.8 & 26,993 & 7.0 \\
\hline N-Acetylleucine_OL2a_6.25_196.0949 m/z & 1666 & 33.7 & 2598 & 42.8 & 877 & 36.9 & 1382 & 48.1 \\
\hline 2-Hydroxyphenylacetate_OL2a_6.32_135.0442 m/z & 7126 & 10.8 & 8264 & 7.3 & 8772 & 4.1 & 8172 & 2.9 \\
\hline 2-Octenedioic Acid_OL2a_6.81_195.0632 m/z & 3387 & 13.2 & 2517 & 12.6 & 2645 & 16.8 & 2972 & 15.3 \\
\hline Dethiobiotin_OL2a_7.37_429.2716 m/z & 201 & 173.2 & 229 & 87.2 & 59,537 & 22.1 & 78,517 & 17.8 \\
\hline 2-Octenedioic Acid_OL2a_7.42_195.0631 m/z & 7730 & 17.3 & 5094 & 9.2 & 3140 & 38.0 & 7516 & 8.4 \\
\hline Suberate_OL2a_7.47_197.0789 m/z & 129,165 & 4.3 & 118,707 & 4.1 & 117,587 & 2.1 & 137,784 & 4.8 \\
\hline Suberate_OL2a_7.6_157.0862 m/z & 23,101 & 11.6 & 23,341 & 6.1 & 19,390 & 8.1 & 20,297 & 13.2 \\
\hline Caprylate_OL2a_9.32_167.1047 m/z & 27,549 & 5.2 & 27,692 & 7.5 & 28,494 & 2.5 & 32,939 & 3.8 \\
\hline Sebacate_OL2a_9.72_225.1102 m/z & 52,739 & 6.7 & 48,714 & 3.8 & 46,481 & 12.6 & 53,830 & 6.9 \\
\hline 10-Hydroxy-2-Decenoic Acid_OL2a_9.84_209.1152 $\mathrm{m} / \mathrm{z}$ & 117,259 & 4.7 & 116,924 & 2.7 & 115,816 & 4.8 & 129,228 & 0.7 \\
\hline 3-Hydroxyadipic 3,6 Lactone_OL2b_0.69_144.0424 $n$ & 34,905 & 7.9 & 31,933 & 9.1 & 56,260 & 19.5 & 64,756 & 13.0 \\
\hline Acetoacetate_OL2b_0.71 $102.0318 n$ & 39,375 & 8.5 & 36,703 & 2.3 & 37,028 & 3.1 & 40,002 & 8.9 \\
\hline Adipate_OL2b_0.8_111.0442 m/z & 34,128 & 1.6 & 34,202 & 5.8 & 33,653 & 4.9 & 35,715 & 0.7 \\
\hline Pyridoxine OL2b $0.8 \quad 169.0741 n$ & 286,515 & 17.4 & 256,692 & 9.7 & 15,455 & 13.7 & 14,008 & 71.9 \\
\hline Pyroglutamic Acid_OL2b_0.82_259.0929 m/z & 11,248 & 38.6 & 10,178 & 24.9 & 0 & - & 0 & - \\
\hline 2-Hydroxybutyrate_OL2b_0.82_87.0442 $\mathrm{m} / \mathrm{z}$ & 8654 & 7.0 & 8841 & 5.6 & 10,005 & 4.0 & 10,868 & 0.3 \\
\hline L-Cysteinylglycine_OL2b_1.29_179.0488 m/z & 157,188 & 14.9 & 137,522 & 22.2 & 194,013 & 8.6 & 266,356 & 10.1 \\
\hline Glutarate_OL2b_1.67_115.0392 m/z & 29,496 & 2.4 & 30,845 & 5.5 & 26,960 & 9.6 & 26,663 & 9.5 \\
\hline 3-Methyladenine_OL2b_1.87_150.0777 m/z & 768 & 22.7 & 429 & 136.3 & 2705 & 66.5 & 1279 & 11.0 \\
\hline Benzoate_OL2b_10.34_105.0337 m/z & 1707 & 5.5 & 1842 & 17.8 & 1346 & 28.5 & 1501 & 48.1 \\
\hline 2-Octenedioic Acid_OL2b_11.17_173.0811 m/z & 0 & - & 0 & - & 1342 & 99.6 & 0 & - \\
\hline 10-Hydroxydecanoic Acid_OL2b_11.39_153.1277 m/z & 9020 & 1.7 & 8179 & 0.7 & 8623 & 8.7 & 8677 & 7.6 \\
\hline 10-Hydroxydecanoic Acid_OL2b_11.42_171.1383 m/z & 11,427 & 0.8 & 10,545 & 5.9 & 10,166 & 7.9 & 10,899 & 8.0 \\
\hline Sphinganine_OL2b_11.92_302.3058 m/z & 54,577 & 7.3 & 55,845 & 2.8 & 53,097 & 6.7 & 57,716 & 2.4 \\
\hline Azelate_OL2b_12.05_153.0912 m/z & 6061 & 7.5 & 6831 & 2.1 & 6209 & 14.8 & 6855 & 5.6 \\
\hline Heptanoic Acid_OL2b_12.45_283.1883 m/z & 48,315 & 4.2 & 46,278 & 5.3 & 45,251 & 2.9 & 52,254 & 8.8 \\
\hline Sphinganine_OL2b_12.47_284.2952 m/z & 2750 & 47.7 & 2659 & 27.2 & 5898 & 38.0 & 7522 & 50.9 \\
\hline 2-Hydroxytetradecanoic Acid_OL2b_12.73_267.1934 m/z & 43,587 & 4.9 & 42,755 & 9.2 & 44,157 & 2.7 & 48,037 & 6.1 \\
\hline Sphinganine_OL2b_12.75_302.3058 m/z & 16,252 & 19.1 & 16,622 & 25.9 & 47,603 & 11.8 & 56,698 & 10.6 \\
\hline
\end{tabular}


Table 2. Cont.

\begin{tabular}{|c|c|c|c|c|c|c|c|c|}
\hline \multirow[b]{2}{*}{ Compound } & \multicolumn{2}{|c|}{ Scrape-Freeze-Thaw } & \multicolumn{2}{|c|}{ Scrape-Homogenize } & \multicolumn{2}{|c|}{ Trypsinize-Homogenize } & \multicolumn{2}{|c|}{ Trypsinize-Freeze-Thaw } \\
\hline & Avg & RSD (\%) & Avg & RSD (\%) & Avg & RSD (\%) & Avg & RSD (\%) \\
\hline Sphinganine_OL2b_12.82_284.2952 $\mathrm{m} / \mathrm{z}$ & 3644 & 18.8 & 4261 & 20.3 & 13,496 & 17.6 & 13,425 & 15.9 \\
\hline Hexadecanoylcarnitine_OL2b_13.1_400.3428 m/z & 16,550 & 7.3 & 15,958 & 4.4 & 19,620 & 11.5 & 25,724 & 12.2 \\
\hline Benzoate_OL2b_13.15_105.0337 m/z & 1858 & 8.3 & 1680 & 1.0 & 1746 & 6.3 & 1752 & 10.5 \\
\hline Linoleic Acid_OL2b_13.19_263.2374 m/z & 9159 & 13.6 & 8004 & 5.8 & 7610 & 11.6 & 8222 & 2.5 \\
\hline Oleoylcarnitine_OL2b_13.27_426.3584 m/z & 20,182 & 12.4 & 18,244 & 24.1 & 17,383 & 14.8 & 24,494 & 10.1 \\
\hline 12-Hydroxy-9-Cis-Octadecenoic Acid_OL2b_13.47_263.2373 m/z & 11,675 & 12.9 & 10,852 & 8.4 & 7759 & 5.0 & 8027 & 8.6 \\
\hline Octadecanoylcarnitine_OL2b_13.67_428.3741 m/z & 22,954 & 10.3 & 22,440 & 12.7 & 26,152 & 20.7 & 34,914 & 22.5 \\
\hline Linoleic Acid_OL2b_13.9_263.2373 m/z & 6141 & 25.8 & 5205 & 14.5 & 1131 & 9.4 & 1722 & 23.7 \\
\hline 2-Hydroxytetradecanoic Acid_OL2b_13.92_267.1933 m/z & 27,633 & 1.3 & 26,690 & 1.8 & 25,513 & 7.7 & 26,874 & 3.6 \\
\hline 12-Hydroxy-9-Cis-Octadecenoic Acid_OL2b_14.28_321.2403 m/z & 494,789 & 4.8 & 560,842 & 2.0 & 561,252 & 4.2 & 532,630 & 6.3 \\
\hline 2-Hydroxyglutarate_OL2b_14.35_297.0827 m/z & 4411 & 17.7 & 6365 & 28.2 & 6406 & 18.4 & 4074 & 8.4 \\
\hline Palmitoleic Acid_OL2b_14.58_254.2248 $n$ & 46,698 & 14.3 & 51,379 & 12.5 & 58,247 & 14.3 & 57,708 & 8.9 \\
\hline Palmitoleic Acid_OL2b_14.79_254.2248 n & 40,233 & 8.1 & 51,315 & 12.9 & 60,231 & 9.3 & 47,499 & 11.6 \\
\hline Eicosapentaenoate_OL2b_14.94_325.2140 m/z & 867 & 10.3 & 15,576 & 58.1 & 11,425 & 42.1 & 711 & 10.4 \\
\hline Docosahexaenoate_OL2b_15.21_328.2403 n & 112,516 & 20.5 & 329,323 & 43.5 & 292,259 & 38.5 & 66,066 & 1.5 \\
\hline 27-Hydroxycholesterol_OL2b_15.37_385.3468 m/z & 31,043 & 45.5 & 23,565 & 34.7 & 33,490 & 23.1 & 43,309 & 45.5 \\
\hline 3-Hydroxy-3-Methylglutaric Acid_OL2b_3.21_145.0498 m/z & 14,199 & 10.1 & 15,513 & 19.1 & 9555 & 24.4 & 9991 & 9.0 \\
\hline B-Nicotinamide Mononucleotide_OL2b_3.28_335.0645 m/z & 976,592 & 7.2 & 928,731 & 15.9 & 776,931 & 8.7 & $1,065,967$ & 12.3 \\
\hline Leucine_OL2b_3.77_154.0842 m/z & 55,154 & 49.7 & 68,733 & 49.4 & 30,573 & 74.7 & 40,142 & 85.4 \\
\hline 10-Hydroxydecanoic Acid_OL2b_3.82_153.1277 m/z & 5351 & 9.9 & 5687 & 21.9 & 6232 & 17.6 & 6492 & 9.0 \\
\hline Glutarate_OL2b_3.89_155.0317 m/z & 5471 & 11.0 & 15,289 & 17.5 & 9519 & 18.6 & 2705 & 19.0 \\
\hline N-Acetylserotonin_OL2b_5.93_219.1133 m/z & 8978 & 6.6 & 9545 & 10.2 & 8470 & 10.4 & 8677 & 18.3 \\
\hline $2^{\prime}, 4^{\prime}$-Dihydroxyacetophenone_OL2b_6.18_153.0549 m/z & 15,564 & 4.1 & 15,019 & 7.5 & 14,038 & 2.1 & 16,217 & 4.9 \\
\hline Sebacate_OL2b_6.57_167.1070 m/z & 9903 & 8.2 & 9679 & 16.8 & 8116 & 19.3 & 8824 & 10.8 \\
\hline Sebacate_OL2b_6.81_167.1070 m/z & 3801 & 13.2 & 3013 & 15.0 & 2798 & 32.3 & 3462 & 11.0 \\
\hline Pantothenate_OL2b_6.98_242.1004 m/z & 4065 & 21.2 & 3328 & 18.3 & 3597 & 9.3 & 3750 & 10.9 \\
\hline Sebacate_OL2b_7.47_203.1282 m/z & 10,163 & 5.2 & 9709 & 4.2 & 9555 & 4.1 & 10,235 & 9.0 \\
\hline Hexanoyl Glycine_OL2b_7.99_196.0948 m/z & 35 & 173.2 & 1665 & 108.1 & 4102 & 59.9 & 116 & 16.6 \\
\hline Benzoate_OL2b_8.62_105.0337 m/z & 1728 & 11.9 & 1440 & 21.0 & 1131 & 34.3 & 1630 & 9.7 \\
\hline Paraxanthine_OL2b_8.65_180.0641 $n$ & 94,367 & 14.5 & 127,026 & 18.4 & 113,606 & 7.2 & 85,535 & 9.1 \\
\hline 10-Hydroxydecanoic Acid_OL2b_9.97_153.1277m/z & 7148 & 2.5 & 7353 & 15.6 & 6869 & 1.4 & 7221 & 4.1 \\
\hline
\end{tabular}

Metabolites are displayed in the following format: compound name_ontology level_retention time_mass. Masses ending with $n$ are neutral masses whereas masses ending in $m / z$ are ion masses. 


\section{Discussion}

In the current study, we set out to investigate how different sample preparation methods affected the types and abundances of metabolites that could be extracted from MDA-MB-231 cells for metabolomics analysis. Two major steps of cell sample preparation were investigated: detachment and lysis methods. Overall, it was observed that the detachment method had the greatest effect on peak abundance and affected a larger number of signals than the lysis methods. Overall, scraped samples had higher peak abundances than trypsinized samples. This observation has been made in other studies using other cell types $[10,11,16,17,20]$. This phenomenon of trypsinization causing a decrease in metabolite abundance has been referred to as "metabolite leakage" and it is thought that trypsin leads to permeabilization of cell membranes, allowing metabolites to leak out of the cell and into the extracellular space [20]. However, it should be noted that a large number of signals were increased in trypsinized samples, which were matched to metabolites related to fatty acid metabolism, such as acylcarnitines. This observation suggests that many of these metabolic differences may arise due to a biological response to the trypsinization process. Typically, cells are incubated with trypsin for $\sim 5-10 \mathrm{~min}$ in order to sufficiently degrade extracellular matrix proteins and complete the detachment process. Indeed, cancer cells have been shown to undergo metabolic adaptations during the dissemination phase of metastasis, indicating that metabolism adapts after the loss of anchorage [21]. Many metabolites that were observed in the trypsinized samples were related to energy utilization (e.g., fatty acids and lactate). Given that metabolism can respond to stressors within seconds [22], it is highly possible that the trypsinization itself can greatly shift the metabotype of cells. Further investigation is needed to understand if these metabolic differences are a consequence of the technique, or if they are part of the biological response to matrix detachment. In contrast, the scraping method allows for the immediate quenching of metabolism with the addition of a cold organic solution directly onto adhered cells, minimizing any metabolic adaptations to occur during the detachment process. This information is of great importance as our data suggest that the metabolic response to these two detachment methods are significantly different. Therefore, not only should researchers consider the implications of these detachment methods on the extraction efficiencies of metabolites, but they should also consider that the metabolism of cells can significantly shift in response to these conditions (such as trypsinization) in a relatively small timeframe, which may change the interpretability of the study results. It should also be noted that differences in matrices, particularly between trypsinized and scraped samples, were minimized through the dilution and washing of trypsinized samples prior to metabolite extraction. The use of a high-organic solution for metabolite extraction (80\% methanol) also served to precipitate any remaining trypsin prior to analysis, further diminishing potential matrix effects.

In contrast to the detachment method, the two lysis methods overall had more subtle differences in metabolic profiles, although they were still distinct in the multivariate analysis. For example, some cholesterols, acylcarnitines, and polyamines (spermine and spermidine) showed large differences between the freeze-thaw and homogenizer bead methods. Although this is a minor effect overall compared to the detachment methods, care should be taken in selecting the best lysis method based on the needs of the study being performed. Using pathway analysis, we were able to find differences in certain metabolic pathways even if certain individual metabolites did not seem to be significantly affected. From this, we were able to see changes at a broader level across the different sample preparation methods. This analysis also showed that the differences between the two detachment methods are much broader than what was seen between the lysis methods (sixteen perturbed pathways vs. four perturbed pathways). Careful consideration should be taken to ensure the best sample preparation method is used depending on the metabolic pathways of interest, particularly those that were identified as significant in our pathway analysis. While other studies have investigated the effects of trypsinization on metabolite classes, ours is the first to show a significant effect on acylcarnitines that were elevated 
following trypsin. This may be due to a specific property of MDA-MB-231 cells, or it could be that other studies did not include acylcarnitines in their metabolomics libraries. Regardless, this poses questions about the role of fatty acid metabolism in response to trypsin or other proteases. Cancer cells are known to undergo metabolic adaptations following matrix detachment, particularly glucose and glutamine metabolism, as a method to combat anoikis and oxidative stress during metastatic processes [21,23]. It is possible that the significant changes in acylcarnitines also indicate that alterations in fatty acid occur during the detachment process, either as a method to supply alternate energy sources or as a method to create antiapoptotic signals to survive the detachment process. Lastly, our findings that many metabolites significantly increase or decrease across these sample preparation methods was also reflected in the entire peak set (Figure 4). This indicates that there are many unknown metabolites, beyond those identified in this manuscript, that are heavily affected by these detachment and lysis methods. This indicates the need for additional studies to further identify this metabolomics "dark matter" to better understand the metabolite classes and pathways affected by these processes.

\section{Materials and Methods}

\subsection{Chemical and Reagents}

All solvents for UHPLC-HRMS analysis (Optima-grade water and methanol with $0.1 \%$ formic acid) and fetal bovine serum (FBS) were purchased from Fisher Scientific (Waltham, MA, USA). Dulbecco's Modified Eagle Medium (DMEM) high glucose and phosphate-buffered saline were purchased from Gibco (Grand Island, NY, USA). Reagents for the bicinchoninic acid (BCA) assays were purchased from Thermo Scientific (Madison, WI, USA). The MDA-MB-231 cell line was purchased from the American Type Culture Collection (ATCC) (Manassas, VA, USA). MagNA lyser tubes with ceramic beads were purchased from Roche Diagnostics (Indianapolis, IN, USA).

\subsection{Cell Culture}

MDA-MB-231 cells were maintained in DMEM high glucose supplemented with 10\% FBS, $2 \mathrm{mM}$ glu- tamine, $50 \mathrm{U} / \mathrm{mL}$ penicillin, and $50 \mu \mathrm{g} / \mathrm{mL}$ streptomycin. Cells were grown in a $37^{\circ} \mathrm{C}, 5 \% \mathrm{CO}_{2}$, humidity-controlled environment. To prepare cells for different extraction methods, $4 \times 10^{6}$ MDA-MB-231 cells were plated into $100 \mathrm{~mm}$ tissue culturetreated dishes and allowed to adhere overnight, achieving approximately $80 \%$ confluency. Blank culture dishes were used for all of the following methods to generate method blanks.

\subsection{Cell Scraping Detachment Method}

Culture dishes containing MDA-MB-231 cells were placed on ice. Media was aspirated and dishes were washed with $5 \mathrm{~mL}$ of cold PBS. After removing the PBS, $2 \mathrm{~mL}$ of ice-cold Homogenization Solution (80-20 methanol-water) was added to each dish for metabolite quenching. Cells were lifted off culture dishes by the use of cell scrapers. For each dish, $500 \mu \mathrm{L}$ of the cell suspension was aliquoted into a tube for freeze-thaw lysis and another $500 \mu \mathrm{L}$ was aliquoted into a tube for bead homogenization.

\subsection{Trypsinization Detachment Method}

To detach cells by trypsinization, the media were aspirated, and dishes were washed with $5 \mathrm{~mL}$ of cold PBS. After removal of PBS, $1 \mathrm{~mL}$ of Trypsin-EDTA $(0.25 \%)$ was added to each dish followed by incubation at $37^{\circ} \mathrm{C}$ for $5 \mathrm{~min}$. Trypsin-EDTA was then neutralized by the addition of $10 \mathrm{~mL} \mathrm{DMEM}$, which was then removed by aspirating the supernatant after centrifuging cell suspensions for $5 \mathrm{~min}$ at $0.9 \mathrm{rpm}$ at $8{ }^{\circ} \mathrm{C}$. Cell pellets were washed with $2 \mathrm{~mL}$ ice-cold PBS and then re-centrifuged using the above parameters to aspirate the PBS. Cell pellets were then resuspended in $2 \mathrm{~mL}$ ice-cold Homogenization Solution. For each cell suspension, $500 \mu \mathrm{L}$ was aliquoted into a tube for freeze-thaw lysis and another $500 \mu \mathrm{L}$ was aliquoted into a tube for bead homogenization. 


\subsection{Bead Homogenization}

As described above, $500 \mu \mathrm{L}$ aliquots of cell suspensions from both the cell scraping and trypsinization detachment methods were placed in MagNa lyser tubes with pre-washed ceramic beads. Tubes were placed onto an Omni Bead Ruptor Elite (OMNI International) at $6.00 \mathrm{~m} / \mathrm{s}$ for 2 cycles at $45 \mathrm{~s}$ each with a $30 \mathrm{~s}$ dwell time in between. Lysates were immediately placed on ice following the homogenization procedure.

\subsection{Freeze-Thaw Cycling}

As described above, $500 \mu \mathrm{L}$ aliquots of cell suspensions from both detachment methods were placed into tubes for freeze-thaw cycling. Tubes were placed into liquid nitrogen for $30 \mathrm{~s}$ for freezing and then placed into a hot water bath set to $37^{\circ} \mathrm{C}$ for $30 \mathrm{~s}$ to thaw. This process was completed a total of three times to facilitate cell lysis. Lysates were immediately placed on ice following the freeze-thaw procedure.

\subsection{Protein Measurement}

Aliquots of $100 \mu \mathrm{L}$ from each sample were dried by speedvac for $2 \mathrm{~h}$ and then redissolved in $400 \mu \mathrm{L}$ of a $5 \%$ sodium dodecyl sulfate (SDS) solution. Protein concentrations for each sample were measured by a BCA assay. Additional Homogenization Solution was added to the remaining $400 \mu \mathrm{L}$ of each sample to normalize the metabolites by protein concentration.

\subsection{UHPLC-HRMS Data Acquisition and Multivariate Statistical Analysis}

Prior to analysis, a total quality control study pool (QCSP) was generated by combining $10 \mu \mathrm{L}$ of each sample. Individual study samples, blanks (LC-MS grade water), and the QCSP were vortexed at $5000 \mathrm{rpm}$ for $10 \mathrm{~min}$ using a multi-tube vortex. Samples were centrifuged at $16,000 \mathrm{rcf}$ for $10 \mathrm{~min}$ at $4{ }^{\circ} \mathrm{C}$, transferred to autosampler vials, and $5 \mu \mathrm{L}$ was injected for analysis. The individual study samples were randomized, and blanks and QCSP injections were interspersed amongst the study sample in the run sequence. Data were acquired using a Vanquish UHPLC system coupled to a Q Exactive ${ }^{\mathrm{TM}} \mathrm{HF}-\mathrm{X}$ Hybrid Quadrupole-Orbitrap Mass Spectrometer (Thermo Fisher Scientific, San Jose, CA, USA) using previously published methods [24-27]. Metabolites were separated via an HSS T3 C18 column $\left(2.1 \times 100 \mathrm{~mm}, 1.7 \mu \mathrm{m}\right.$, Waters Corporation) at $50{ }^{\circ} \mathrm{C}$ with a binary mobile phase of water (A) and methanol (B), each containing $0.1 \%$ formic acid $(v / v)$. The UHPLC linear gradient started from $2 \% \mathrm{~B}$, and increased to $100 \% \mathrm{~B}$ in $16 \mathrm{~min}$, then held for $4 \mathrm{~min}$, with the flow rate at $400 \mu \mathrm{L} / \mathrm{min}$. Data-dependent acquisition of the untargeted data was acquired from 70 to $1050 \mathrm{~m} / \mathrm{z}$ in positive mode. Peak picking, alignment, and normalization was performed using Progenesis QI (version 2.1, Waters Corporation). Peaks that had a higher average abundance in the blanks as compared to the QCSP were filtered out of the dataset. Principal Component Analysis (PCA) and Orthogonal Partial Least SquaresDiscriminant Analysis (OPLS-DA) was performed on the normalized, preprocessed data using SIMCA 16 (Sartorius Stedim Data Analytics AB, Umeå, Sweden). Unit Variance (UV) scaling was used for all multivariate plots.

\subsection{Pathway Analysis}

Pathway analysis was performed using MetaboAnalyst 5.0 [28] to identify metabolic pathways that were significantly altered across the sample preparation methods. All peaks from the normalized, preprocessed data (4479) were input into the "Functional Analysis" module as a peak intensity table and grouped based upon their detachment and lysis method. A mass tolerance of $5 \mathrm{ppm}$ was used, and the retention time information was included for each peak. Pathway significance was calculated using the default top $10 \%$ of peaks by $p$-value (ANOVA, calculated by MetaboAnalyst) with the Homo sapiens (human) [MFN] option selected as the pathway library. Both the mummichog and GSEA algorithms were selected to generate pathway scatter plots. Additionally, a heatmap of all peaks, across 
all groups, was generated using the "Functional Analysis" module of MetaboAnalyst to compare peak abundance profiles across each extraction method.

\subsection{Metabolite Identification}

Metabolites were identified in Progenesis QI using an in-house physical standards library of reference standards. All reference standards were analyzed under the same conditions used to analyze the study samples. Peaks were matched to metabolites in the in-house library by exact mass (MS), fragmentation pattern (MS/MS), and retention time (RT). An ontology system was provided for each peak match to indicate the evidence basis for each identification. A peak was considered to have a match by RT if the peak eluted within 0.5 min compared to the reference standard; an MS match was defined as $<5 \mathrm{ppm}$ error compared to the theoretical mass based on the metabolite chemical formula; and an MS/MS match was defined as a similarity score $\geq 30$ to a reference standard (calculated using MS/MS match algorithms in Progenesis QI). OL1 refers to an in-house metabolite match by RT, MS, and MS/MS; OL2a refers to a match by RT and MS only; and OL2b refers to a match by MS and MS/MS only.

\subsection{Statistical Analysis of Identified Metabolites}

Metabolites that were matched to the in-house library by an OL1, OL2a, or OL2b definition were input into the "Statistical Analysis" module in MetaboAnalyst as an intensity table using abundance values from the normalized, preprocessed dataset. Heatmaps and One-way Analysis of Variance (ANOVA) modules in MetaboAnalyst were used to determine the changes in these metabolites across the detachment and lysis methods.

\section{Conclusions}

In conclusion, detachment and lysis methods produce significantly different metabolic profiles (in terms of the number of features detected, and their abundance) in MDA-MB-231 cells. Overall, scraped samples had a higher abundance of metabolites although it should be noted that a subset of metabolites increased in trypsinized samples. These increased metabolites may be due to enhanced yield, or they may be a consequence of the biological response to matrix detachment. Interestingly, this is an observation that was seen in another study using HeLa and MCF-7 cells [20]. As such, great care must be taken when selecting trypsinization as a detachment method for metabolomics studies, as some of the metabolic changes may be influenced by the acute metabolic response to proteolytic release from the extracellular matrix, such as fatty acid metabolism, as seen by the significant increases in acylcarnitines in trypsinized samples. The difference between lysis methods was less pronounced, with both freeze-thaw cycling and bead homogenization producing similar results for most metabolites. These results provide a better understanding for how different sample preparation procedures affect extracted metabolite profiles, which will aid in future studies investigating the metabolism of triple-negative breast cancer cells. Furthermore, this study shows how MDA-MB-231 cells-a highly common model for triple-negative breast cancer-responds to various commonly used sample preparation methods. Future studies are needed to determine if these trends are also observed in other cell models.

Supplementary Materials: The following supporting information can be downloaded at: https: / / www.mdpi.com/article/10.3390/metabo12020168/s1, Supplemental Table S1. Metabolite statistics across QC pool injections.

Author Contributions: Study concept and design-B.R.R. and S.C.J.S.; data acquisition-B.R.R. and M.S.; statistical analysis-B.R.R.; identification/annotation of metabolites-B.R.R. and M.S.; data interpretation-All; manuscript preparation-B.R.R.; revision of manuscript drafts-All. All authors have read and agreed to the published version of the manuscript.

Funding: This work was supported by the Nutrition Obesity Research Center (P30DK056,350-21).

Institutional Review Board Statement: Not applicable. 


\section{Informed Consent Statement: Not applicable.}

Data Availability Statement: The data presented in this study are available on request from the corresponding author due to privacy restrictions.

Conflicts of Interest: The authors have no conflict of interest to disclose.

\section{References}

1. DeBerardinis, R.J.; Chandel, N.S. Fundamentals of cancer metabolism. Sci. Adv. 2016, 2, e1600200. [CrossRef] [PubMed]

2. Rahman, M.; Hasan, M.R. Cancer metabolism and drug resistance. Metabolites 2015, 5, 571-600. [CrossRef] [PubMed]

3. Boroughs, L.K.; DeBerardinis, R.J. Metabolic pathways promoting cancer cell survival and growth. Nat. Cell Biol. 2015, 17, 351-359. [CrossRef]

4. Wong, C.C.; Qian, Y.; Yu, J. Interplay between epigenetics and metabolism in oncogenesis: Mechanisms and therapeutic approaches. Oncogene 2017, 36, 3359-3374. [CrossRef]

5. Levine, A.J.; Puzio-kuter, A.M. The control of the metabolic switch in cancer by oncogenes and tumor suppressor genes. Science 2010, 330, 1340-1344. [CrossRef]

6. Liu, X.; Locasale, J.W. Metabolomics-A primer. Trends Biochem. Sci. 2017, 42, 274-284. [CrossRef] [PubMed]

7. Sumner, L.W.; Amberg, A.; Barrett, D.; Beale, M.H.; Beger, R.; Daykin, C.A.; Fan, T.W.M.; Fiehn, O.; Goodacre, R.; Griffin, J.L.; et al. Proposed minimum reporting standards for chemical analysis: Chemical Analysis Working Group (CAWG) Metabolomics Standards Initiative (MSI). Metabolomics 2007, 3, 211-221. [CrossRef]

8. Herman, S.; Emami Khoonsari, P.; Aftab, O.; Krishnan, S.; Strömbom, E.; Larsson, R.; Hammerling, U.; Spjuth, O.; Kultima, K.; Gustafsson, M. Mass spectrometry based metabolomics for in vitro systems pharmacology: Pitfalls, challenges, and computational solutions. Metabolomics 2017, 13, 79. [CrossRef]

9. Peterson, A.L.; Walker, A.K.; Sloan, E.K.; Creek, D.J. Optimized method for untargeted metabolomics analysis of MDA-MB-231 breast cancer cells. Metabolites 2016, 6, 30. [CrossRef]

10. Bi, H.; Krausz, K.W.; Manna, S.K.; Li, F.; Johnson, C.H.; Gonzalez, F.J. Optimization of harvesting, extraction, and analytical protocols for UPLC-ESI-MS-based metabolomic analysis of adherent mammalian cancer cells. Anal. Bioanal. Chem. 2013, 405, 5279-5289. [CrossRef]

11. Dettmer, K.; Nürnberger, N.; Kaspar, H.; Gruber, M.A.; Almstetter, M.F.; Oefner, P.J. Metabolite extraction from adherently growing mammalian cells for metabolomics studies: Optimization of harvesting and extraction protocols. Anal. Bioanal. Chem. 2011, 399, 1127-1139. [CrossRef] [PubMed]

12. Muschet, C.; Möller, G.; Prehn, C.; de Angelis, M.H.; Adamski, J.; Tokarz, J. Removing the bottlenecks of cell culture metabolomics: Fast normalization procedure, correlation of metabolites to cell number, and impact of the cell harvesting method. Metabolomics 2016, 12, 151. [CrossRef] [PubMed]

13. Lorenz, M.A.; Burant, C.F.; Kennedy, R.T. Reducing time and increasing sensitivity in sample preparation for adherent mammalian cell metabolomics. Anal. Chem. 2011, 83, 3406-3414. [CrossRef] [PubMed]

14. Ibáñez, C.; Simó, C.; Palazoglu, M.; Cifuentes, A. GC-MS based metabolomics of colon cancer cells using different extraction solvents. Anal. Chim. Acta 2017, 986, 48-56. [CrossRef] [PubMed]

15. Danielsson, A.P.H.; Moritz, T.; Mulder, H.; Spégel, P. Development and optimization of a metabolomic method for analysis of adherent cell cultures. Anal. Biochem. 2010, 404, 30-39. [CrossRef] [PubMed]

16. Kapoore, R.V.; Coyle, R.; Staton, C.A.; Brown, N.J.; Vaidyanathan, S. Influence of washing and quenching in profiling the metabolome of adherent mammalian cells: A case study with the metastatic breast cancer cell line MDA-MB-231. Analyst 2017, 142, 2038-2049. [CrossRef]

17. Kapoore, R.V.; Coyle, R.; Staton, C.A.; Brown, N.J.; Vaidyanathan, S. Cell line dependence of metabolite leakage in metabolome analyses of adherent normal and cancer cell lines. Metabolomics 2015, 11, 1743-1755. [CrossRef]

18. Kathryn, J.C.; Sireesha, V.G.; Stanley, L. Triple Negative Breast Cancer Cell Lines: One Tool in the Search for Better Treatment of Triple Negative Breast Cancer. Breast Dis. 2012, 32, 35-48. [CrossRef]

19. Triba, M.N.; Le Moyec, L.; Amathieu, R.; Goossens, C.; Bouchemal, N.; Nahon, P.; Rutledge, D.N.; Savarin, P. PLS/OPLS models in metabolomics: The impact of permutation of dataset rows on the K-fold cross-validation quality parameters. Mol. Biosyst. 2015, 11, 13-19. [CrossRef]

20. Luo, X.; Gu, X.; Li, L. Development of a simple and efficient method of harvesting and lysing adherent mammalian cells for chemical isotope labeling LC-MS-based cellular metabolomics. Anal. Chim. Acta 2018, 1037, 97-106. [CrossRef]

21. Endo, H.; Owada, S.; Inagaki, Y.; Shida, Y.; Tatemichi, M. Metabolic reprogramming sustains cancer cell survival following extracellular matrix detachment. Redox Biol. 2020, 36, 101643. [CrossRef] [PubMed]

22. Wilson, D.F. Regulation of cellular metabolism: Programming and maintaining metabolic homeostasis. J. Appl. Physiol. 2013, 115, 1583-1588. [CrossRef] [PubMed]

23. Paoli, P.; Giannoni, E.; Chiarugi, P. Anoikis molecular pathways and its role in cancer progression. Biochim. Biophys. Acta-Mol. Cell Res. 2013, 1833, 3481-3498. [CrossRef] 
24. Rushing, B.; McRitchie, S.; Arbeeva, L.; Nelson, A.; Azcarate-Peril, M.; Li, Y.; Qian, Y.; Pathmasiri, W.; Sumner, S.; Loeser, R. Fecal metabolomics reveals products of dysregulated proteloysis and altered microbial metabolism in obesity-related osteoarthritis. Osteoarthr. Cartil. 2022, 30, 81-91. [CrossRef] [PubMed]

25. Li, S.; Li, Y.; Rushing, B.R.; Harris, S.E.; McRitchie, S.L.; Sumner, S.J.; Dohlman, H.G. Multi-omics analysis of multiple glucosesensing receptor systems in yeast. Biomolecules 2022, 12, 175. [CrossRef]

26. Li, Y.-Y.; Ghanbari, R.; Pathmasiri, W.; McRitchie, S.; Poustchi, H.; Shayanrad, A.; Roshandel, G.; Etemadi, A.; Pollock, J.D.; Malekzadeh, R.; et al. Untargeted Metabolomics: Biochemical Perturbations in Golestan Cohort Study Opium Users Inform Intervention Strategies. Front. Nutr. 2020, 7, 2020-2021. [CrossRef]

27. Li, S.; Li, Y.; Rushing, B.R.; Harris, S.E.; McRitchie, S.L.; Jones, J.C.; Dominguez, D.; Sumner, S.J.; Dohlman, H.G. Multi-omics analysis of glucose-mediated signaling by a moonlighting G $\beta$ protein Asc1/RACK1. PLoS Genet. 2021, 17, e1009640. [CrossRef]

28. Pang, Z.; Chong, J.; Zhou, G.; De Lima Morais, D.A.; Chang, L.; Barrette, M.; Gauthier, C.; Jacques, P.É.; Li, S.; Xia, J. MetaboAnalyst 5.0: Narrowing the gap between raw spectra and functional insights. Nucleic Acids Res. 2021, 49, 388-396. [CrossRef] 